\title{
SUR LE NOMBRE DE SINGULARITÉS DÉGÉNÉRÉES D'UNE FAMILLE DE FEUILLETAGES
}

\author{
ISRAEL VAINSENCHER
}

RÉSumÉ. Nous présentons des formules pour le nombre des singularités dégénérés (resp. nilpotentes) des membres d'un pinceau (resp. réseau) général de feuilletages de dimension un dans une variété (resp. surface) projective lisse.

\begin{abstract}
A César Camacho, à l'occasion de son 60 ème anniversaire.
\end{abstract}

\section{Introduction}

La recherche des invariants numériques attachés à des feuilletages algébriques remonte à Poincaré [12], dans le cadre de la détermination des bornes pour le degré de courbes invariantes dans $\mathbb{C P}^{2}$. Les travaux plus récents traitent cette question en établissant des relations pour les nombres de singularités du feuilletage et certains nombres de Chern, tout en profitant des arguments de positivité [2], [5], [6], [7], [13].

Soit $\mathcal{F}$ un feuilletage de dimension un et degré $k$ dans $\mathbb{C P}^{n}$, c'est-à-dire une section holomorphe non nulle $\sigma: \mathcal{O} \rightarrow T \mathbb{C P}^{n} \otimes \mathcal{O}(k-1)$ (à un multiple scalaire prés). Les singularités de $\mathcal{F}$ sont les zéros de $\sigma$. Soit $\mathcal{S}=\mathcal{S}_{\mathcal{F}}$ l'ensemble des singularités de $\mathcal{F}$. On sait que quand $\mathcal{S}$ est finie, il contient $1+k+\cdots+k^{n}$ points, comptés avec des multiplicités naturelles. Si $\mathcal{F}$ est choisi général dans $\mathbb{P}\left(H^{0}\left(T \mathbb{C P}^{n} \otimes \mathcal{O}(k-1)\right)\right)$ alors $\mathcal{S}$ est fini et toutes les multiplicités sont égales à un.

Nous considérons d'abord la question de trouver le nombre de singularités dégénérées qu'on espére apparaître dans un pinceau général de feuilletages.

Théoréme A. Soit $\mathcal{S}^{1}$ le schéma de singularités dégénérés dans un pinceau de feuilletages à fibré cotangent $L$ au-dessus d'une variété lisse projective $M$. Si $\mathcal{S}^{1}$ est fini, on a

$$
\operatorname{deg}\left(\mathcal{S}^{1}\right)=n \sum_{0}^{n}(n+1-i) \int_{M} c_{i} \cdot H^{n-i},
$$

où on note $n=\operatorname{dim} M, H=c_{1} L, c_{i}=c_{i} T M$.

\footnotetext{
Received September 29, 2003.

2000 Mathematics Subject Classification: 14N10 (Primary); 14C20, 14H40, 14K05 (Sec-

Key words and phrases. intersection theory, singularities, foliations.

Travail partiellement soutenu par CNPq-Brèsil.
} ondary). 

$k$.

Pour $M=\mathbb{C P}^{n}$ on obtient $(n+1) k^{n}$ pour un pinceau de feuilletages de degré

Si on a plus de paramètres à notre disposition (e.g., un réseau de feuilletages), alors on espère trouver en général, une sous-famille de dimension (au moins) un de feuilletages ayant une singularité dégénérée qui bouge avec le feuilletage. On peut alors imposer des conditions sur la partie linéaire du feuilletage à la singularité. Par exemple, pour des surfaces, on espère trouver un nombre fini de singularités dégénérées à partie linéaire nilpotente. Nous donnons une formule en termes des classes de Chern de la surface et du fibré cotangent du feuilletage.

Théoréme B. Soit $M$ une surface projective lisse et soit $\mathbb{T}$ un réseau de feuilletages à fibré cotangent $L$. Soit $\mathcal{S}^{2}$ l'ensemble de pairs $(t, x) \in \mathbb{T} \times M$ tels que $x$ est un point singulier de $\mathcal{F}_{t}$ à partie linéaire nilpotente. Si $\mathcal{S}^{2}$ est fini, alors il consiste de

$$
\operatorname{deg}\left(\mathcal{S}^{2}\right)=2 \int_{M}\left(c_{2}+3 c_{1} \cdot H+6 H^{2}\right)
$$

points comptés avec des multiplicités naturelles, où $H=c_{1} L, c_{i}=c_{i} T M$.

Pour $\mathbb{C P}^{2}$, la formule se réduit à $6\left(2 k^{2}-1\right)$, où $k$ dénote le degré du feuilletage.

\section{Préliminaires}

Soit $E \rightarrow M$ un fibré vectoriel au-dessus d'une variété lisse et projective $M$. On note $r$ le rang de $E$.

\subsection{Le schéma d'incidence. Soit}

$$
V \subset H^{0}(M, E)
$$

un sous-espace de l'espace des sections globales. On a au-dessus de $\mathbb{P}(V) \times M$ le diagramme d'homomorphismes de fibrés vectoriels,

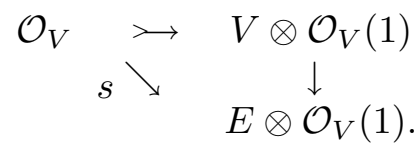

La flèche horizontale provient de la suite exacte d'Euler au-dessus de $\mathbb{P}(V)$. La section notée $s$ est dite la section d'évaluation universelle pour la famille de sections dans $V$. Pour chaque point $t \in \mathbb{P}(V)$, la restriction $s_{t}$ de $s$ à $\{t\} \times M \simeq$ $M$ s'identifie à une section de $E$ (à multiplication par scalaire non nul prés).

On considère, pour chaque sous-schéma $\mathbb{T} \subseteq \mathbb{P}(V)$, le schéma d'incidence, défini par l'idéal image de la co-section $s^{\star}:\left(E \otimes \mathcal{O}_{V}(1)\right)^{\star} \rightarrow \mathcal{O}_{\mathbb{T}}$ au-dessus de $\mathbb{T}$,

$$
\mathbb{P}(V) \times M \supseteq \mathcal{S}_{\mathbb{T}}=\left\{(t, x) \in \mathbb{T} \times M \mid s_{t}(x)=0\right\}
$$




\subsection{Lemme. Notations comme ci-dessus,}

1. si $\mathbb{T}$ est de dimension pure alors chaque composante irréductible du schéma d'incidence est de codimension $\geq r$;

2. si E est engendré par sections globales et $V$ est général, alors on a l'égalité $\operatorname{codim} \mathcal{S}_{\mathbb{P}(V)}=r$

3. si $\mathbb{T}$ est de Cohen-Macaulay et $\mathcal{S}_{\mathbb{T}}$ est vide ou de la bonne codimension, on a la formule

$$
\left[\mathcal{S}_{\mathbb{T}}\right]=c_{r}\left(E \otimes \mathcal{O}_{V}(1)\right) \cap[\mathbb{T} \times M]
$$

dans le groupe de Chow $A^{r}(\mathbb{T} \times M)$ des cycles de codimension $r$.

Démonstration. Soit $U=H^{0}(M, E)$. Supposons $E$ engendré par sections globales, donc on a la suite exacte

$$
W \succ U \times M \rightarrow E .
$$

Le fibré $W$ est de la bonne codimension $r$ dans le fibré trivial $U \times M$. On voit tout de suite que $\mathcal{S}_{\mathbb{P}(U)}$ s'identifie à $\mathbb{P}(W) \subseteq \mathbb{P}(U) \times M$. L'image réciproque d'un sous-schéma $\mathbb{T}$ de $\mathbb{P}(U)$ par l'application $\mathbb{P}(W) \rightarrow \mathbb{P}(U)$ induite par projection s'identifie à $\mathcal{S}_{\mathbb{T}}$. L'affirmation (2) découle de Bertini [10, p. 89]; pour les autres affirmations, voir [8], Lemme A.7.1, p. 418 et Prop. 14.1, p. 244.

Nous laissons au soin du lecteur la vérification de qu'on peut considérer aussi la situation où on se donne une famille de fibrés vectoriels au-dessus de variétés lisses, c'est-à-dire, une application $\mathbb{M} \rightarrow \mathbb{T}$ lisse et une section d'un fibré vectoriel $E$ au-dessus de $\mathbb{M}$.

2.3. Parties principales. On rappelle le fibré des parties principales, $\mathcal{P}_{M}^{1}(E)$ [4, p. 14]. La fibre au-dessus de $x \in M$ est l'espace vectoriel

$$
\mathcal{P}_{M}^{1}(E)_{x}=\left(\mathcal{O}_{M, x} / \mathfrak{m}_{x}^{2}\right) \otimes E_{x}
$$

où $\mathfrak{m}_{x} \subset \mathcal{O}_{M, x}$ dénote l'idéal des fonctions nulles en $x$. Étant donné un germe de fonction $f \in \mathcal{O}_{M, x}$, l'image $\bar{f}$ dans $\mathcal{O}_{M, x} / \mathfrak{m}_{x}^{2}$ est son jet d'ordre un, c'est-à-dire, l'expansion de Taylor tronquée tout en gardant la valeur $f(x)$ aussi bien que les dérivées du premier ordre. On a par construction la suite exacte,

$$
\Omega_{M}^{1} \otimes E \succ \mathcal{P}_{M}^{1}(E) \rightarrow E .
$$

Chaque section $\sigma: \mathcal{O} \rightarrow E$ se relève à une section $\sigma^{1}: \mathcal{O} \rightarrow \mathcal{P}_{M}^{1}(E)$ rendant commutatif le diagramme

$$
\begin{array}{ccc}
\mathcal{O}_{M} & & \\
\sigma^{1} \downarrow & \searrow \sigma & \\
\mathcal{P}_{M}^{1}(E) & \rightarrow & E .
\end{array}
$$

Quand $\sigma_{x}$ s'annule, il s'en suit que $\sigma_{x}^{1}$ se factorise par le noyau $\left(\Omega_{M}^{1} \otimes E\right)_{x}$. La flèche induite dans ce noyau n'est autre que la jacobienne de $\sigma$ au point $x$, via l'identification habituelle $\Omega_{M}^{1} \otimes E \simeq \operatorname{Hom}(T M, E)$.

Mettant ensemble $(\star)$ et $(\diamond)$ on montre aisément le suivant. 
2.4. Lemme. Notations comme ci-dessus, on a le diagramme d'homomorphismes de fibrés vectoriels

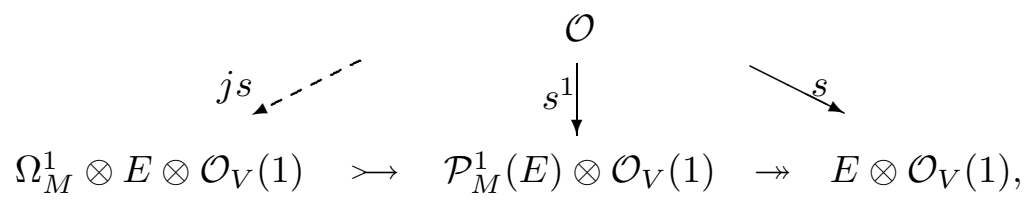

où la flèche brisée existe aprés restriction du diagramme au-dessus du schéma de zéros $\mathcal{S}$ de $s$.

2.5. Dégénérations. On se pose désormais au-dessus de $\mathcal{S}$, le schéma de zéros de la section $s$. On a la section jacobienne

$$
j s: T M \longrightarrow E \otimes \mathcal{O}_{V}(1)
$$

Alors on peut imposer des conditions de rang sur $j s$. La formule de Porteous (cf. [8, p. 254]) nous permet d'obtenir un tas de relations numériques pour les classes de Chern. Le cas d'intérêt ici est quand on prend $r=\operatorname{rang} E=n=\operatorname{dim} M$.

Étant donné un sous-schéma $\mathbb{T} \subseteq \mathbb{P}\left(H^{0}(E)\right)$, nous définissons le schéma de dégénérations $\mathcal{S}^{1} \subseteq \mathcal{S}$ comme le schéma de zéros de $\wedge^{n} j s: \wedge^{n} T M \rightarrow \wedge^{n}\left(E \otimes \mathcal{O}_{V}(1)\right)$.

$$
\mathcal{S}^{1}=\left\{(t, x) \in \mathbb{T} \times M \mid(t, x) \in \mathcal{S}_{\mathbb{T}} \text { avec } \operatorname{det}\left(j s_{t}(x)\right)=0\right\} .
$$

Prenons d'abord $\mathbb{T}=\mathbb{C P}^{1}$, c'est-à-dire, un pinceau.

2.6. Proposition. Notations comme ci-dessus, si $\mathcal{S}^{1}$ est fini alors on a

$$
\operatorname{deg}\left(\mathcal{S}^{1}\right)=\int_{M}\left(\left(c_{1} E+K\right) \cdot c_{n-1} E+n c_{n} E\right)
$$

où $K=c_{1} \Omega_{M}^{1}$.

Démonstration. Écrivons $h=c_{1} \mathcal{O}_{V}(1)$, la classe hyperplane de $\mathbb{C P}^{1}$. Nous avons

$$
\begin{array}{ccc}
c_{n}\left(E \otimes \mathcal{O}_{V}(1)\right) & c_{n} E+h \cdot c_{n-1} E, \\
c_{1}\left(\wedge^{n}\left(E \otimes \mathcal{O}_{V}(1)\right) \otimes \wedge\right. & \left.\Omega_{M}^{1}\right) & \left(c_{1} E+n h+K\right) .
\end{array}
$$

Si $\mathcal{S}$ est vide ou de la bonne dimension (un), on peut écrire le cycle

$$
[\mathcal{S}]=c_{n}\left(E \otimes \mathcal{O}_{V}(1)\right) \cap[\mathbb{T} \times M]
$$

dans $A_{1}(\mathbb{T} \times M)$. Quand $\mathcal{S}=\emptyset$, il vient $c_{n}\left(E \otimes \mathcal{O}_{V}(1)\right)=0$. On déduit que

$$
0=\left(c_{1} E+K+n h\right) \cdot\left(c_{n} E+h \cdot c_{n-1} E\right)=\left(\left(c_{1} E+K\right) \cdot c_{n-1} E+n c_{n} E\right) \cdot h(\bullet)
$$

donc le coefficient de $h$ est nul aussi et la formule est trivialement vraie. Supposons maintenant $\mathcal{S} \neq \emptyset$. Dans ce cas là, toutes les composantes irréductibles de $\mathcal{S}$ ont la bonne dimension un, sinon $\mathcal{S}^{1}$, étant défini dans $\mathcal{S}$ par une équation locale, ne serait pas fini. On écrit d'abord

$$
\left[\mathcal{S}^{1}\right]=\left(c_{1} E+n h+K\right) \cap[\mathcal{S}]
$$

dans $A^{1}(\mathcal{S})$. Ceci entraîne

$$
\left[\mathcal{S}^{1}\right]=\left(c_{1} E+K+n h\right) \cdot\left(c_{n} E+h c_{n-1} E\right)=\left(\left(c_{1} E+K\right) \cdot c_{n-1} E+n c_{n} E\right) \cdot h
$$


dans $A^{1}\left(\mathbb{C P}^{1} \times M\right)$ en vue de la derniére expression dans $(\bullet)$. On finit en appliquant la formule de projection.

On applique ce que précède à

$$
E=T M \otimes L
$$

(et de même $M=\mathbb{C P}^{n}$ ) avec un fibré en droites $L$. Donc les sections donnent lieu à des feuilletages. On appelle $L$ le fibré cotangent du feuilletage défini par une section non nulle de $T M \otimes L$. Si $L$ est assez ample, alors pour un pinceau général $V \subseteq H^{0}(E)$, le schéma de singularités $\mathcal{S}$ est de la bonne dimension (voir 3.3) et le lieu de dégénérations $\mathcal{S}^{1}$ est fini. On arrive ainsi à la formule suivante pour son degré.

2.7. Corollaire. Soit $\mathcal{S}^{1}$ le schéma de singularités dégénérés dans un pinceau de feuilletages à fibré cotangent $L$ au-dessus d'une variété lisse projective $M$. Si $\mathcal{S}^{1}$ est fini, on a

$$
\operatorname{deg}\left(\mathcal{S}^{1}\right)=n \sum_{0}^{n}(n+1-i) \int_{M} c_{i} \cdot H^{n-i}
$$

où on note $n=\operatorname{dim} M, H=c_{1} L, c_{i}=c_{i} T M$.

Démonstration. Nous avons $c_{1}(T M \otimes L+K)=-K+n H+K$, donc

$$
\begin{aligned}
\operatorname{deg}\left(\mathcal{S}^{1}\right) & =\int c_{1}(T M \otimes L+K) \cdot c_{n-1}(T M \otimes L)+n c_{n}(T M \otimes L) \\
& =n \int H \sum_{0}^{n-1}(n-i) c_{i} \cdot H^{n-1-i}+n \sum_{0}^{n} c_{i} \cdot H^{n-i} .
\end{aligned}
$$

2.8. Exemples. (1) Soit $M$ une courbe projective lisse de genre $g$ et soit $L$ un fibré en droites de degré $d$. Alors $E=T M \otimes L$ est de degré $2-2 g+d$. La formule pour $\operatorname{deg}\left(\mathcal{S}^{1}\right)$ donne $2(d+1-g)$. C'est le nombre des diviseurs dans un pinceau générique de $L \otimes K^{\star}$ ayant un point double.

(2) $M=\mathbb{C P}^{n}$. On a $E=T M \otimes L=T \mathbb{C P}^{n} \otimes \mathcal{O}(k-1)$. Alors, notant $H=c_{1} \mathcal{O}_{\mathbb{C P}^{n}}(1)$, et rappelant la classe de Chern totale, $c T \mathbb{C P}^{n}=(1+H)^{n+1}$, nous pouvons écrire,

$$
\operatorname{deg}\left(\mathcal{S}^{1}\right)=n \sum_{0}^{n}(n+1-i)\left(\begin{array}{c}
n+1 \\
i
\end{array}\right)(k-1)^{n-i}=(n+1) k^{n} .
$$

Pour $n=1=k$, il s'agit d'un pinceau de champs vectoriels dans $\mathbb{C P}^{1}$, c'est-àdire, un pinceau de formes binaires quadratiques. On sait bien qu'il-y-a alors deux membres à racine double.

(3) $M$ une variété abélienne de dimension $n$. Alors le fibré tangent $T M=\mathcal{O}^{n}$ est trivial, donc $c_{i}(T M)=0 \forall i \neq 0$. On prends un fibré en droites $L$ trés ample. On écrit $H=c_{1} L, d=\operatorname{deg}\left(H^{n}\right)$, et on calcule,

$$
\operatorname{deg}\left(\mathcal{S}^{1}\right)=n(n+1) d .
$$


Si on prend $n=1, M$ plongée dans $\mathbb{C P}^{2}$ comme une cubique lisse et $L=\mathcal{O}(1)_{\mid M}$, on retrouve le nombre de droites tangentes passant par un point.

\section{3. plus dégénération}

Nous considérons maintenant un cas de singularités d'ordre supérieur posé par A. Lins et L.G. Mendes à l'IMPA, à la jolie occasion de la fête césarienne. Prenons une surface $M$. On s'intéresse aux feuilletages qui présentent une singularité d'expression locale,

$$
\text { (\$) }\left\{\begin{aligned}
\dot{x} & =u(x, y)=y+\cdots \\
\dot{y} & =v(x, y)=\cdots
\end{aligned}\right.
$$

où $\cdots$ est nul mod. $\langle x, y\rangle^{2}$. Ça veut dire que la partie linéaire peut se mettre sous la forme $\left(\begin{array}{ll}0 & 1 \\ 0 & 0\end{array}\right)$. D'une façon invariante, on demande que la partie linéaire soit nilpotente (en général $\neq 0$ ). En termes de la jacobienne $j s: T M \rightarrow T M \otimes L$, on peut faire la composition

$$
T M \stackrel{j s}{\longrightarrow} T M \otimes L \stackrel{j s \otimes L}{\longrightarrow} T M \otimes L \otimes L .
$$

Mais attention! Il faut prendre garde de ne pas imposer carrément la condition

$$
(j s \otimes L) \circ j s=0 .
$$

En effet, cette composition est une section du fibré de rang 4,

$$
\operatorname{Hom}\left(T M, T M \otimes L^{\otimes 2}\right)
$$

(au-dessus de $\mathcal{S}$ ). Par contre, dans l'espace de matrices $2 \times 2$, le lieu des matrices nilpotentes est de codimension deux, étant donné par les équations det $=0=$ trace. De même, on peut montrer que l'idéal

$$
\left\langle x_{1}^{2}+x_{2} x_{3}, x_{1} x_{2}+x_{2} x_{4}, x_{1} x_{3}+x_{3} x_{4}, x_{2} x_{3}+x_{4}^{2}\right\rangle
$$

engendré par les entrés du carré de la matrice $2 \times 2$ générique $\left(\begin{array}{ll}x_{1} & x_{2} \\ x_{3} & x_{4}\end{array}\right)$ n'est pas réduit: son radical est en fait l'idéal (premier) engendré par

$$
x_{1}+x_{4}(=\text { trace }), x_{1} x_{4}-x_{2} x_{3}(=\mathrm{det}) .
$$

3.1. Lemme. Soit $E$ un fibré de rang deux et soit $L$ un fibré en droites au dessus d'un schéma $X$ de Cohen-Macaulay et de dimension pure. Soit $\alpha: E \rightarrow E \otimes L$ un homomorphisme de rang $\geq 1$ partout. Soit $\mathcal{S}^{2}$ l'ensemble des points $x$ dans $X$ telles que $(\alpha \otimes L) \circ \alpha=0$ au-dessus de $x$. Alors $\mathcal{S}^{2}$ est muni d'une structure naturelle de sous-schéma fermé et chaque composante est de codimension au moins deux. Si la codimension est égale à deux, on a la formule pour le cycle

$$
\left[\mathcal{S}^{2}\right]=2\left(c_{1} L\right)^{2} \cap[X]
$$

dans $A^{2} X$.

Démonstration. L'affirmation sur la codimension vient des équations locales det $=0=$ trace déjà citées. On impose d'abord ${ }^{\wedge} \wedge \alpha=0$; soit $Y$ le sous-schéma ainsi défini. L'hypothèse de rang au moins 1 entraîne (cf. le lemme 3.2 ci-dessous) 
l'existence d'un sous-fibré $F \subset E$ au-dessus de $Y$, de rang un, tel que $\alpha_{Y}$ se factorise par $F \otimes L$. Voilà le diagramme:

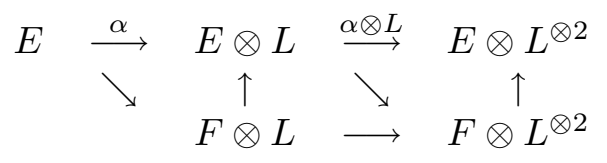

tout au-dessus de $Y$. Notant que $\stackrel{2}{\wedge} \alpha$ est une section de $\stackrel{2}{\wedge} E^{\star} \otimes \stackrel{2}{\wedge} E \otimes L^{\otimes 2} \simeq L^{\otimes 2}$, on a $[Y]=2 c_{1} L \cap[X]$. La condition de nilpotence s'exprime maintenant par l'annulation de la flèche inférieure, $F \otimes L \longrightarrow F \otimes L^{\otimes 2}$. Ce-là se traduit par $\left[\mathcal{S}^{2}\right]=c_{1} L \cap[Y]=2\left(c_{1} L\right)^{2} \cap[X]$, comme énoncé.

3.2. Lemme. Soient E, $G$ des fibrés vectoriels au dessus d'un schéma $Y$. Soit $\alpha: E \rightarrow G$ un homomorphisme de rang $r$ partout. Alors l'image $F$ de $\alpha$ est un sous-fibré (localement scindé) de G.

Démonstration. L'affirmation est locale, donc on se raméne à la situation suivante. Soit $A$ un anneau local et soit $m=\left(m_{i j}\right), m_{i j} \in A$ une matrice de rang $r$ partout. L'hypothése de rang constant $r$ signifie que l'idéal de Fitting engendré par les mineurs $r \times r$ est égal à $A$ tandis que $\wedge^{r+1} m=0$. Alors on peut trouver des matrices inversibles $p, q$ à coefficients dans $A$ telles que $p m q$ soit de la forme $\left(\begin{array}{ll}I & 0 \\ 0 & 0\end{array}\right)$ avec $I$ un bloc identité $r \times r$. (C'est un cas particulier de [11, Lemma 1, p. 233].)

Étant établi que les singularités du type $(\mathbf{A})$ imposent deux conditions, on en espère un nombre fini pour un réseau général $\mathbb{C P}^{2} \subseteq \mathbb{P}\left(H^{0}(M, T M \otimes L)\right)$.

Soit $M$ une variété lisse projective. Soit $L$ un fibré en droites. Nous dirons que une famille $\mathbb{T} \subseteq \mathbb{P}\left(H^{0}(M, T M \otimes L)\right)$ de feuilletages à fibré cotangent $L$ est réguliére si chaque schéma de singularités $\mathcal{S}_{\mathbb{T}}^{2} \subset \mathcal{S}_{\mathbb{T}}^{1} \subseteq \mathcal{S}_{\mathbb{T}}$ a la bonne codimension si non vide.

Mettons au point le fait de que, en général, les choses marchent bien pour la codimension.

3.3. Lemme. Soit $M$ une variété lisse projective. Soit $L$ un fibré en droites ample. Alors il-y-a un entier $m_{0}$ tel que pour tout $m \geq m_{0}$ la famille de feuilletages donnée par un choix de sous-espace général de $\mathbb{P}\left(H^{0}\left(M, T M \otimes L^{\otimes m}\right)\right.$ est réguliére.

Démonstration. Rappelons la construction des fibrés de parties principales [4]. Soient $p_{i}: M \times M \rightarrow M$ les projections $(i=1,2)$. Soit $\mathcal{I}$ le faisceau d'idéaux définissant la diagonale $\Delta \subset M \times M$. Alors $\mathcal{I}^{2}$ définit l'idéal de la voisinage infinitésimal $2 \Delta$. On a le diagramme de suites exactes de faisceaux,

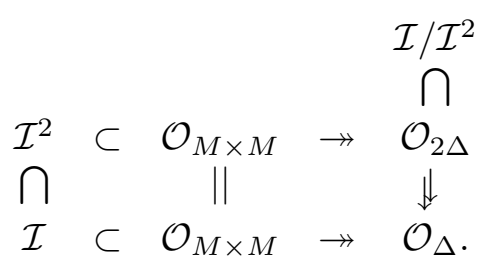


On sait que $\mathcal{I} / \mathcal{I}^{2}$ est le faisceau des sections du fibré cotangent $\Omega_{M}^{1}$ ([8, p. 435], en rappelant que le cotangent est le conormal du plongement diagonal). On se donne un fibré vectoriel $E \rightarrow M$ (on prendra $E=T M \otimes L$ dans un instant). Soient $\mathcal{E}, \mathcal{L}$ les faisceaux de sections de $E, L$ (c'està-dire, $E=\operatorname{Spec}\left(\operatorname{Sym}\left(\mathcal{E}^{\vee}\right)\right)$ comme dans $\left[8\right.$, B.5.5 p.434]). On tensorise le diagramme ci-dessus par $p_{2}^{\star}(\mathcal{E} \otimes \mathcal{L})$ et on applique $\left(p_{1}\right)_{\star}$. Le conoyau $R^{1} p_{1 \star}\left(\mathcal{I}^{2} p_{2}^{\star}(\mathcal{E} \otimes \mathcal{L})\right)$ de la flèche $p_{1 \star} p_{2}^{\star}(\mathcal{E} \otimes$ $\mathcal{L}) \rightarrow p_{1 \star}\left(\mathcal{O}_{2 \Delta} \otimes p_{2}^{\star}(\mathcal{E} \otimes \mathcal{L})\right)$, ainsi que les autres images directes superieures, s'annulent, quitte à remplacer $\mathcal{L}$ par $\mathcal{L}^{\otimes m}, m>>0([9$, Thm. 8.8(c), p. 252] ou $[3,(2.4 .1)$ (ii) p. 107]). Il en résulte un diagramme de faisceaux localement libres. Soit $U=H^{0}(M, E \otimes L)=H^{0}(M, \mathcal{E} \otimes \mathcal{L})$. On sait que $p_{1 \star} p_{2}^{\star}(\mathcal{E} \otimes \mathcal{L})=U \times M$ $([3,(1.4 .15)$ p. 92$])$. Le diagramme correspondant des fibrés vectoriels associés au-dessus de $M$ s'écrit,

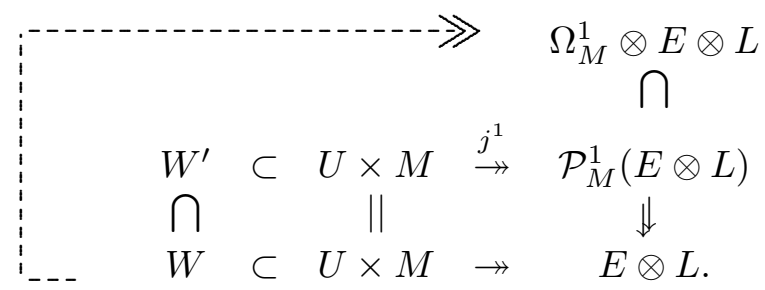

On voit que $W^{\prime}$ est le sous-fibré des pairs $(u, x) \in U \times M$ telles que le jet $j^{1} u$ est nul au point $x$. Comme d'habitude, le lemme du serpent entraîne un homomorphisme surjectif $W \rightarrow \Omega_{M}^{1} \otimes E \otimes L$ de noyau $W^{\prime}$. Si on prend la projectivisation, on a la fibration (donc submersion)

$$
\mathbb{P}(W) \backslash \mathbb{P}\left(W^{\prime}\right) \longrightarrow \mathbb{P}\left(\Omega_{M}^{1} \otimes E \otimes L\right)=\mathbb{P}(\operatorname{Hom}(T M, E \otimes L)) .
$$

Alors l'image réciproque de n'importe quelle sous-variété de $\mathbb{P}(\operatorname{Hom}(T M, E \otimes L))$ conserve la codimension ainsi que les propriétés d'être réduite ou irréductible, etc. On sait bien que les sous-variétés de $\mathbb{P}(\operatorname{Hom}(T M, E \otimes L))$ définies par des conditions de rang sont irréductibles et de la bonne dimension: voir [1, (1.1) p. 5] pour le cas $\operatorname{Hom}(T M, E \otimes L)$; le passage au cas projectif est immédiat. En prenant $E=T M$, si $V \subseteq U$ est un sous-espace assez général avec $\mathbb{T}:=\mathbb{P}(V)$ disjoint de l'image de $\mathbb{P}\left(W^{\prime}\right)$ dans $\mathbb{P}(U)$, il vient que $\mathbb{T}$ est régulier.

3.4. Théoréme B. Soit $M$ une surface projective lisse et soit $\mathbb{T}$ un réseau de feuilletages à fibré cotangent $L$. Soit $\mathcal{S}^{2}$ l'ensemble de pairs $(t, x) \in \mathbb{T} \times M$ tels que $x$ est un point singulier de $\mathcal{F}_{t}$ à partie linéaire locale nilpotente $(\mathbf{A})$. Si $\mathcal{S}^{2}$ est fini, alors il consiste en

$$
\operatorname{deg}\left(\mathcal{S}^{2}\right)=2 \int_{M}\left(c_{2}+3 c_{1} \cdot H+6 H^{2}\right)
$$

points comptés avec des multiplicités naturelles, où $H=c_{1} L, c_{i}=c_{i} T M$.

Démonstration. Le raisonnement est comme dans la preuve de 2.6. 
3.5. Exemples. (1) Pour $M=\mathbb{C P}^{2}$, la formule se réduit à $6\left(2 k^{2}-1\right)$, où $k$ dénote le degré du feuilletage (i.e., $L=\mathcal{O}(k-1)$ ).

(2) Pour une surface abélienne, on trouve $\operatorname{deg}\left(\mathcal{S}^{2}\right)=12 \int H^{2}$. En particulier, pour les bien connues surfaces plongées dans $\mathbb{C P}^{4}$ et feuilletage à fibré cotangent $\mathcal{O}(1)$, on a $\int H^{2}=10$, ce que fournit 120 points dans le cycle $\mathcal{S}^{2}$, tous à crier pour une interprétation classique.

\section{Bibliographie}

[1] W. Bruns, \& U.Vetter, Determinantal rings. Lecture Notes in Mathematics, 1327. Springer-Verlag, Berlin, 1988.

[2] D. Cerveau \& A. Lins Neto, Holomorphic foliations in $\mathbb{C P}^{2}$ having an invariant algebraic curve, Ann. Inst. Fourier 41 (1991), 883-903.

[3] A. Grothendieck \& J. Dieudonné, Éléments de Géométrie Algébrique III-1, Publ. Math. IHES, 11 (1961), 5-167.(aussi dans http://www.numdam.org)

[4] _ Éléments de Géométrie Algébrique IV-4, Publ. Math. IHES, 32 (1967), 5-361.

[5] E. Esteves, The Castelnuovo-Mumford regularity of an integral variety of a vector field on projective space, Math. Research Letters, 9 (2002), 1-15.

[6] E. Esteves \& S. Kleiman, Bounds on leaves of one-dimensional foliations, Bull. Soc. Bras. Matemática, 34 (2003), 145-169.

[7] E. Esteves \& S. Kleiman, Bounding solutions of Pfaff equations. Comm. in Algebra, 31 (2003), 3771-3993.

[8] W. Fulton, Intersection theory, Springer-Verlag, New York, 1985.

[9] R. Hartshorne, Algebraic geometry. Graduate Texts in Mathematics, 52 Springer-Verlag, New York-Heidelberg, 1977.

[10] J.P. Jouanolou, Théorémes de Bertini et Applications, 42, Birkhauser, Boston, 1983.

[11] D. Laksov, The geometry of complete linear maps, Ark. Mat. 26 (1988), 231-263.

[12] H. Poincaré, Sur l'intégration algébrique des équations différentielles du premier ordre et du premier degré, Rend. Circ. Mat. Palermo 5, (1891), 161-191.

[13] M.G. Soares, Projective varieties invariant by one-dimensional foliations, Ann. of Math., 152 (2000), 369-382.

Departamento de Matemática, UFMG-ICEX, Belo Horizonte 30123-970 MG Brasil E-mail address: israel@mat.ufmg.br 